\title{
Recombinant NY-ESO-1 protein with ISCOMATRIX adjuvant induces broad antibody responses in humans, a RAYS-based analysis
}

\author{
AXEL MISCHO $^{1 *}$, NINA BUBEL ${ }^{2 *}$, JONATHAN S. CEBON ${ }^{3}$, PANAGIOTIS SAMARAS ${ }^{1}$, ULF PETRAUSCH $^{1}$, \\ FRANK STENNER-LIEWEN ${ }^{1}$, NIKLAUS G. SCHAEFER ${ }^{1}$, BORIS KUBUSCHOK ${ }^{2}$, \\ CHRISTOPH RENNER ${ }^{1 *}$ and ANDREAS WADLE ${ }^{1 *}$ \\ ${ }^{1}$ Universtity Hospital Zurich, Department of Oncology, CH-80091 Zurich, Switzerland; \\ ${ }^{2}$ University Hospital Homburg, Department Internal Medicine, D-66421 Homburg/Saar, Germany; \\ ${ }^{3}$ Ludwig Institute for Cancer Research, Austin Hospital, Melbourne 3084, Australia
}

Received February 11, 2011; Accepted April 21, 2011

DOI: $10.3892 /$ ijo.2011.1032

\begin{abstract}
Antibody responses to tumor antigens play an important role in initiating a cellular antitumor response with respect to antigen cross-presentation and T cell cross-priming. Successful vaccination strategies rely on an optimally timed activation of the humoral and cellular immune system by using appropriate adjuvant stimulation. The LUD99-008 trial used the cancer testis antigen NY-ESO-1 formulated with ISCOMATRIX adjuvant injected into patients intramuscularly. It was shown that this vaccination strategy is a safe and highly potent activator of the humoral and cellular immune system. Using the RAYS technology, we analyzed in detail the humoral immune response in these patients before and after vaccination: during the course of repeated vaccinations with the adjuvant, antibody titers against NY-ESO-1 and cross-reactivity to LAGE $1 \mathrm{~A}$ and $\mathrm{B}$ increased as an indicator of an enhanced immune response, whereas no antibody response could be detected after vaccination without the adjuvant. Analysis of single fragments of the NY-ESO-1 protein revealed that the humoral response was almost exclusively directed against the $\mathrm{N}$-terminal fragments and the number of fragments and their length being recognized by the NY-ESO-1-specific antibodies increased during the course of vaccination. The humoral immune response mainly consisted of antibodies of the IgG1
\end{abstract}

Correspondence to: Dr Axel Mischo, University Hospital Zurich, Department of Oncology, Rämistr. 100, CH-8091 Zurich, Switzerland E-mail: axel.mischo@usz.ch

*Contributed equally

Abbreviations: CTA, cancer/testis antigen; SEREX, serological analysis of antigens by recombinant cDNA expression cloning; RAYS, recombinant antigen expression on yeast surface

Key words: NY-ESO-1, ISCOMATRIX, antibody, IgG-subclass, recombinant antigen expression on yeast surface and IgG3 subclass. We rarely found $\mathrm{IgG} 2$ and $\mathrm{IgG} 4$ subclass antibodies. Our results support the implication that targetspecific antibodies raised after vaccination contribute to the stimulation of an effective $\mathrm{T}$ cell response against the target antigen.

\section{Introduction}

Besides surgery, chemotherapy and radiation therapy, immunotherapy has emerged in the past decade as supplementary approach to fight cancer (1). Due to its specificity and efficacy, immunotherapy ideally targets the tumor selectively and spares normal tissue. A prerequisite in the field of cancer immunotherapy is the identification and characterization of appropriate target antigens for therapeutic approaches. In modern tumor serology, the powerful SEREX-technology (serological identification of antigens by recombinant expression cloning) allowed for the identification of a multitude of new cancer antigens in various tumor entities by screening of tumor-derived gene expression libraries with high-titered IgG antibodies obtained from the sera of cancer patients. The international SEREX database (www2.licr.org/Cancer ImmunomeDB/) by now holds $>2000$ different gene entries of potentially cancer related antigens. One group of antigens, the so-called cancer/testis antigens (CTAs) has gained special interest among the characterized tumor-specific antigens, as they are only expressed in the immunologically privileged germline cells and various types of human cancers but not in normal tissue (2-4). Of particular interest in this regard is the CTA NY-ESO-1. It is the most immunogenic CTA discovered so far and frequently elicits spontaneous humoral and cellular immune responses in patients with NY-ESO-1 expressing tumors (5).

The SEREX system used for antigen identification is a bacterial expression system not capable of post-translationally modifying recombinantly expressed proteins. Therefore, potential antigens that are subject to post-translational modifications may remain undetected. As a consequence, we established a eukaryotic cDNA expression system in yeast 
(RAYS) (6) with the advantage that recombinant proteins can be expressed on the yeast surface as part of the cell wall in a more naturally folded and partially glycosylated manner $(7,8)$. We and others successfully used this system for serological tumor antigen characterization (6,9-12).

However, the importance of these serological antigen screening technologies goes beyond the serological identification of potential tumor antigens by creating a link between $\mathrm{T}$ and $\mathrm{B}$ cell immunology, as strong antibody responses are considered to depend on the presence of $\mathrm{T}$ cell help $(13,14)$. In vitro, antibody-target complexes greatly increase crosspresentation and induce antigen-specific $\mathrm{CD} 8^{+} \mathrm{T}$ cells (15). Moreover, there are compelling data from clinical trials indicating that target-specific antibodies raised after vaccination are pivotal for target uptake into dendritic cells and crosspresentation to elicit a potent specific $\mathrm{CD} 8^{+} \mathrm{T}$ cell response (16).

The amount and quality of vaccine-induced antibodies depends to a large extent on the adjuvant used for vaccination. The new adjuvant ISOMATRIX, a saponin-based adjuvant, which is known to induce strong antibody- and $\mathrm{T}$ cell responses upon vaccination, was used in a placebo controlled trial with the NY-ESO-1 protein (17). Using the RAYS technology and patient sera from this NY-ESO-1/ISCOMATRIX vaccine trial, we analyzed in detail the humoral responses against NY-ESO-1 and the highly homologous CTA LAGE-1A and LAGE-1B as well as other CTA such as SSX2, SSX4, CT-7 and CT-10 before and after vaccination. ISCOMATRIX seems to be able to raise a durable Th1 type immune response by eliciting a broad antibody response contributing to an efficient cross-presentation and priming of $\mathrm{CD}^{+}$targetspecific T cells. Our results underline the important role of an appropriate antibody response against the target antigen.

\section{Materials and methods}

Expression of cancer testis antigens on yeast surface. The amplified codogen regions of the CT-antigens NY-ESO-1, NY-ESO-1 (trunc.), LAGE-1A, LAGE-1B, SSX-2, SSX-4, CT-7 and CT-10 and the respective NY-ESO-1 fragments were cloned into the multiple cloning site of the pYD1 plasmid (Invitrogen, Leiden, The Netherlands) as described previously $(6,10)$. Plasmids were introduced into Saccharomyces cerevisiae EBY100, using a commercially available transformation kit (EasyComp, Invitrogen), according to the manufacturer's recommendations. Thereafter, yeast was spread on MD-Leu plates $(0.67 \%$ yeast nitrogen base, $2 \%$ dextrose, $0.01 \%$ leucine, $1.5 \%$ agar) for the selection of transformed clones. Colonies were picked and cultured [in YPD/CAA-Glu: $1 \%$ yeast extract, $2 \%$ polypeptone and $2 \%$ dextrose or in YNB/CAA-Glu: $2 \%$ dextrose, $0.67 \%$ yeast nitrogen base and $0.5 \%$ casamino acids (Difco, Livonia, MN, USA)] with constant agitation to an approximate $\mathrm{OD}(600 \mathrm{~nm})$ of $4\left(30^{\circ} \mathrm{C}, 4 \mathrm{~h}\right)$. To induce surface protein expression, yeast were pelleted by centrifugation, resuspended to an OD $(600 \mathrm{~nm})$ of 0.6 in YNB/CAA-Gal and grown with agitation for additional $48 \mathrm{~h}\left(20^{\circ} \mathrm{C}\right)$.

Immunofluorescence staining for flow cytometry. Recombinant antigen expression on yeast surface (RAYS) was used for serological analysis as described (6). In brief, 104-105 transformed and induced yeast cells containing the DNA fragment-pYD1 plasmids were collected by centrifugation ( $2000 \mathrm{x} \mathrm{g}, 5 \mathrm{~min})$, washed with PBS and incubated with $100 \mu$ of pre-absorbed serum (1:100 dilution, RT, $30 \mathrm{~min})$ with occasional agitation. Parental control yeast transformed with empty pYD1 vector served for human sera pre-absorption. Then, cells were washed, secondary biotinylated anti-human-IgG Fc $\gamma$-specific serum (Dianova, Hamburg, Germany) was added and the mixture incubated was with occasional agitation (dilution 1:200, RT, $30 \mathrm{~min})$. Finally, R-phycoerythrin-conjugated streptavidin (Dianova) was added (dilution of 1:200, RT, $15 \mathrm{~min}$ ) and washed twice with PBS ( $0.1 \%$ Tween-20) before analyzing the sera reactivity by flow cytometry (FACScan; BectonDickinson, Heidelberg, Germany). For IgG subclass analysis, anti-human IgG 1-4 subclass-specific secondary antibodies (dilution of 1:200; the binding site, Schwetzingen, Germany) and a tertiary biotinylated rabbit anti-sheep antibody (dilution of 1:200; Jackson Immuno Research) and R-phycoerythrinconjugated strepatvidin was used for detection as described above. Expres-sion control for the cloned antigens was performed by the detection of expressed His-tag, using the specific biotinylated anti-penta-His antibody (1:500, Qiagen, Hilden, Hamburg). Thirty thousand cells were counted and the ratio between the intensity of the signal measured on antigen expressing and non-expressing control yeast was calculated for each individual sample.

Western blot analysis. Verification of yeast surface-expressed protein was done by standard Western blot analysis as described previously (10). Briefly, proteins were cleaved under reducing conditions $\left(100 \mathrm{mM}\right.$ mercaptoethanol, $\left.1 \mathrm{~h}, 20^{\circ} \mathrm{C}\right)$ and concentrated by chloroform/methanol precipitation. Protein $(2-10 \mu \mathrm{g})$ was separated by polyacrylamide gel-electrophoresis. Cell lysates were diluted 1:5 (v/v) in loading buffer [10\% (w/v) SDS, $250 \mathrm{mM}$ Tris $\mathrm{HCl}(\mathrm{pH} 5-6.8)$, $500 \mathrm{mM}$ dithiothreitol, $0.5 \%$ bromophenol blue, $50 \%$ glycerol, $4 \%$ mercaptoethanol] and boiled for 5 min. Proteins were separated on a $12 \%$ SDS-PAGE (running buffer: $25 \mathrm{mM}$ Tris, $250 \mathrm{mM}$ glycine, $0.1 \%$ SDS) at $200 \mathrm{~V}$. Separated proteins were transferred onto PDVF membranes (Millipore, Darmstadt, Germany) in blotting buffer (30 mM glycine, $4 \mathrm{mM}$ Tris-base, $0.037 \%$ SDS, $20 \%$ methanol, pH 5-8.3) for $20 \mathrm{~min}(20 \mathrm{~V}$, 300-350 mA). Proteins were detected by standard procedures. In brief, PDVF membranes were blocked in PBS plus $1 \%$ milk-powder $\left(37^{\circ} \mathrm{C}, 1 \mathrm{~h}\right)$. After 4 washes with PBS, the membrane was incubated with primary antibody [RT 5 30-45 min; anti-c-myc-antibody (9E10), 1:500 (Santa Cruz)]. The secondary antibody [RT 5 30-45 min; 1:2,000 goat antimouse-HRP (Dako, Hamburg, Germany)] was conjugated with horseradish-peroxidase and detected by chemiluminescence (ECL-Kit; Pharmacia, Freiburg, Germany).

Patient sera. The sera from 46 patients originating from the ISCOMATRIX-NY-ESO-1 vaccination trial (LUD99-008) (17) were collected as part of the study. Blood samples were collected with written informed consent. This trial was a placebo-controlled phase I clinical trial evaluating the safety and immunogenicity of recombinant NY-ESO-1 protein with ISCOMATRIX adjuvant. Forty-six evaluable patients with resected NY-ESO-1-positive tumors (mainly melanoma with only minimal or no tumor residue) were vaccinated three times 
A

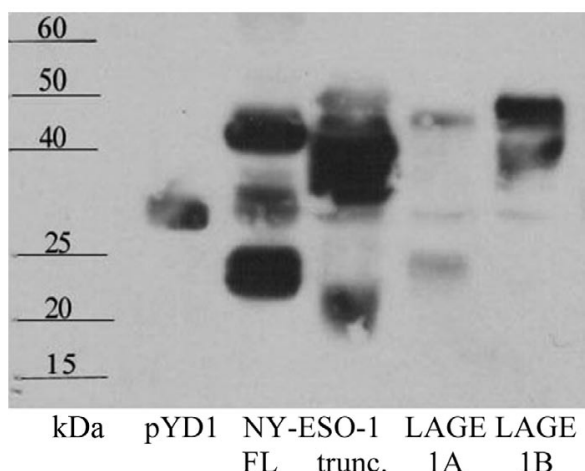

B

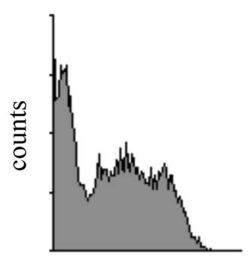

NY Eso FL

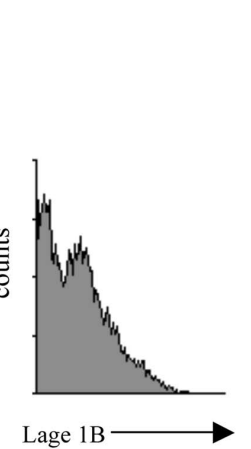

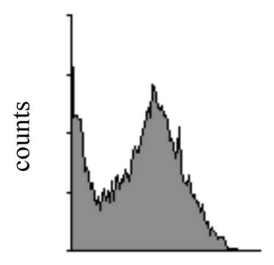
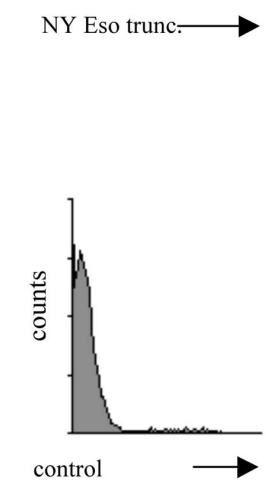

Figure 1. Detection of CT-antigen expression by Western blotting and FACS. (A) Western blotting of CT-antigens cleaved off the yeast cell wall. CT antigens were cleaved off the yeast surface under reducing conditions after induction of expression. Supernatant was used for SDS-PAGE and Western blotting. Proteins were detected via C-terminal Myc-tag. (B) Correct induction of protein expression and yeast cell surface display. Yeast cell flow cytometry histograms after staining the C-terminal Penta-His-tag show the correct display of full length NY-ESO-1, truncated NY-ESO-1, LAGE-1A, LAGE-1B and not induced yeast cells as control.

i.m. every four weeks with different amounts of NY-ESO-1 protein with or without ISCOMATRIX adjuvant.

\section{Results}

CT-antigen expression on yeast surface and recognition by human sera. The serological immune response was assessed at different time points. Altogether, 230 serum samples were analyzed by using the previously described RAYS (6) (recombinant antigen expression on yeast surface) technology, a derivative serological method of the SEREX technology (18). Serological responses to the following CT-antigens were examined: NY-ESO-1 full-length (FL) (AA 1-180), NY-ESO-1 truncated (AA 1-134), LAGE-1A (FL), LAGE-1B (FL). $\mathrm{CT}$-antigens or fragments thereof were cloned into the pYD1expression vector and after yeast transformation surface expression of proteins induced. Protein expression was confirmed after protein cleavage from the yeast surface under reducing
A
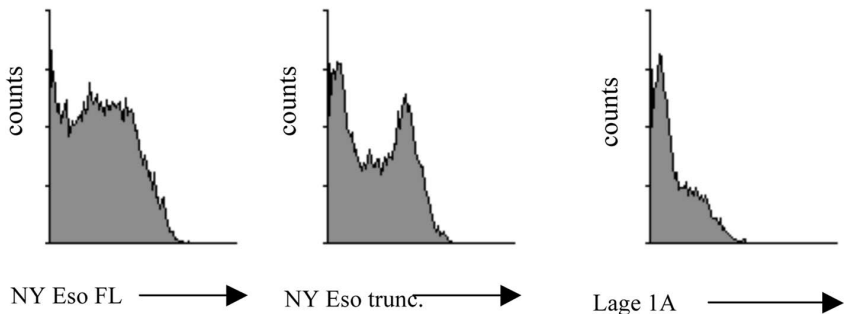

NY Eso FL

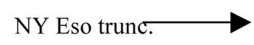

Lage 1A
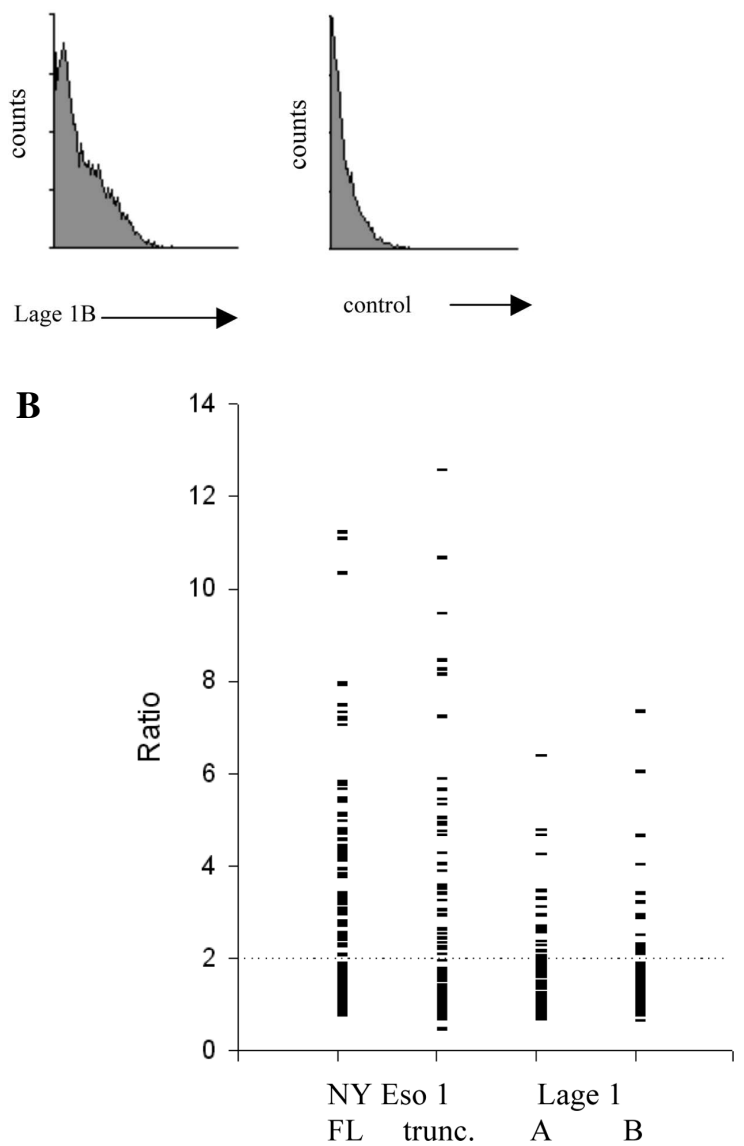

Figure 2. Patient serum antibody responses against NY-ESO-1 FL, NY-ESO-1 truncated, LAGE 1A, LAGE 1B. (A) Flow cytometry, positive patient serum no. 107-H-H-280201: Histograms on full length NY-ESO-1 (FL), truncated NY-ESO-1, LAGE-1A and LAGE-1B and a control. (B) Antibody responses on NY-ESO-1 FL, NY-ESO-1 truncated, LAGE 1A, LAGE 1B. Y-axis, ratio of flow cytometry g-mean values of antigen expressing to empty vector expressing yeast cells. Each dash represents the ratio of an individual serum staining on the indicated antigen expressing yeast cells. Values below the threshold 2 (dotted line) were considered negative.

conditions. The supernatant containing the soluble protein was used for SDS-PAGE and Western blotting. Detection of proteins and confirmation of correct expression was possible due to the C-terminal Myc-tag (Fig. 1A). Due to heavy glycosylation of yeast proteins, the molecular weight of the detected proteins was higher than the calculated weight.

After culturing the yeast cells in glucose-containing medium, protein expression and -display was induced in galactose containing medium. The correct induction was confirmed via anti-Penta-His-tag staining by flow cytometry (Fig. 1B). After 
A

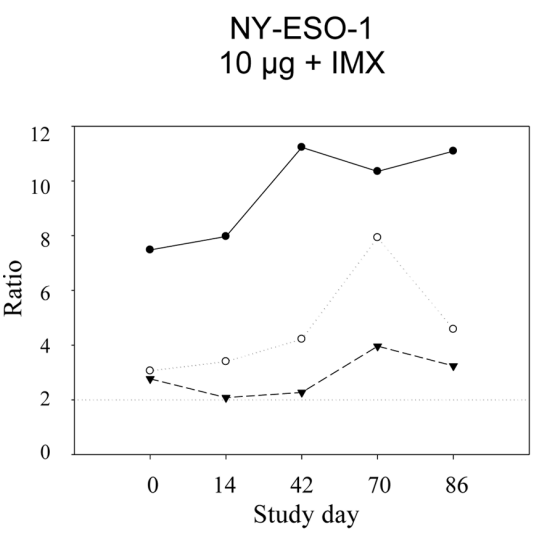

C

NY-ESO-1

$100 \mu \mathrm{g}+\mathrm{IMX}$

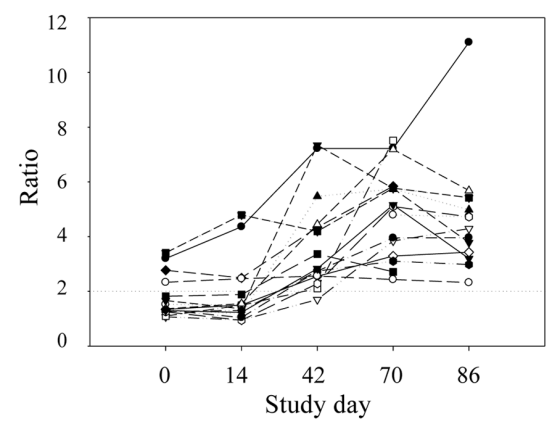

$\mathbf{E}$

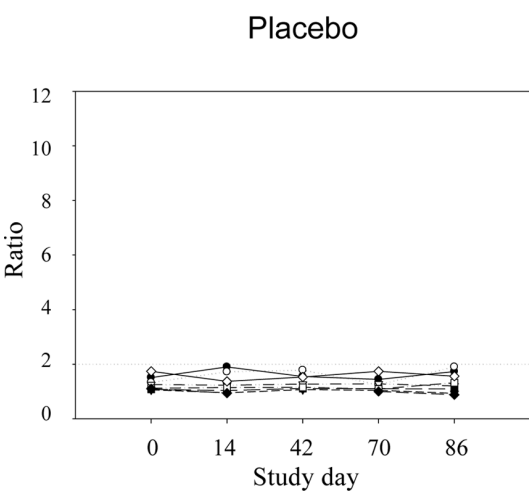

B

NY-ESO-1

$30 \mu \mathrm{g}+\mathrm{IMX}$

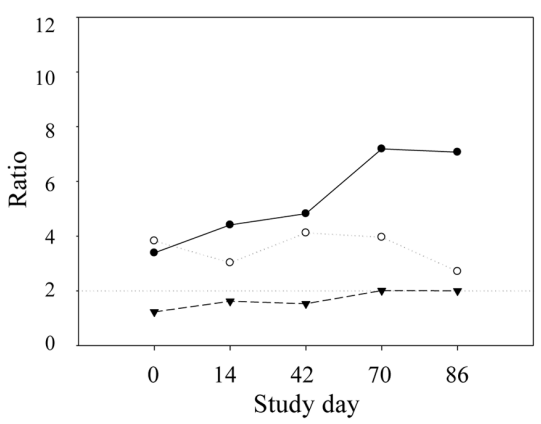

D

$100 \mu \mathrm{g}$, no IMX

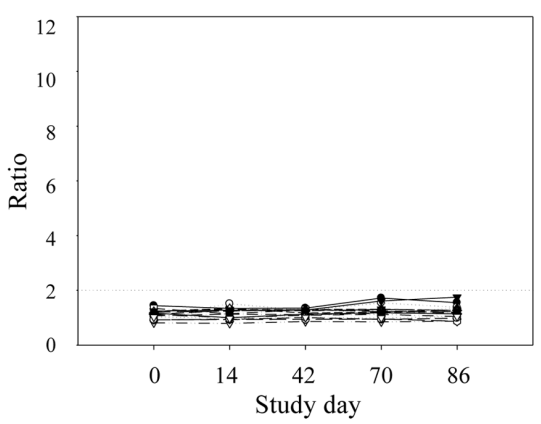

Figure 3. Time course of the immune reaction against NY-ESO-1 FL. Cohort (A) $10 \mu \mathrm{g}$ protein + ISCOM, (B) $30 \mu \mathrm{g}$ protein + ISCOM, (C) $100 \mu \mathrm{g}$ protein + ISCOM, (D) $100 \mu \mathrm{g}$ protein without ISCOM, (E) placebo.

induction, yeast cells were incubated with pre-absorbed patient sera. Bound antigen-specific human IgG was detected via a secondary biotinylated anti-human IgG antibody and streptavidin-PE in FACS. When the ratio of the mean fluorescence intensity (g-mean) of CT-antigen expressing yeast versus empty vector expressing yeast was $>2$, a serum was considered positive. This cut-off point was successfully established in our previous studies $(6,9,10)$ as well as by other groups (12). A positive serum reactivity is shown in Fig. 2A.

Pattern of immune response. The first 10 patients (49 sera) were additionally tested for spontaneous antibody-responses to the CT-antigens SSX-2, SSX-4, CT-7 and CT-10. There were only six positive sera for SSX-2, none for SSX-4, CT-7 and CT-10. Due to this poor response, no further testing for additional CT-antigens was performed (data not shown).

There were 78 sera positive for NY-ESO-1 FL, 40 for NY-ESO-1 trunc., 22 for LAGE-1A and 18 for LAGE-1B (Fig. 2B). We only found a positive reaction on NY-ESO-trunc., LAGE-1A and B, when an immune response to NY-ESO-1 was detectable at the same time.

Consistent with expectations, serum samples were only positive from the patient cohorts $\mathrm{A}, \mathrm{B}$ and $\mathrm{C}$, i.e. the groups that had received the vaccine in combination with the adjuvant ISCOM. No serum reaction could be detected from the cohorts $\mathrm{D}$ i.e. the group with protein vaccination without adjuvant and E (placebo). Fig. 3 shows the time course of the antibody response against NY-ESO-1 FL in the patient 
A

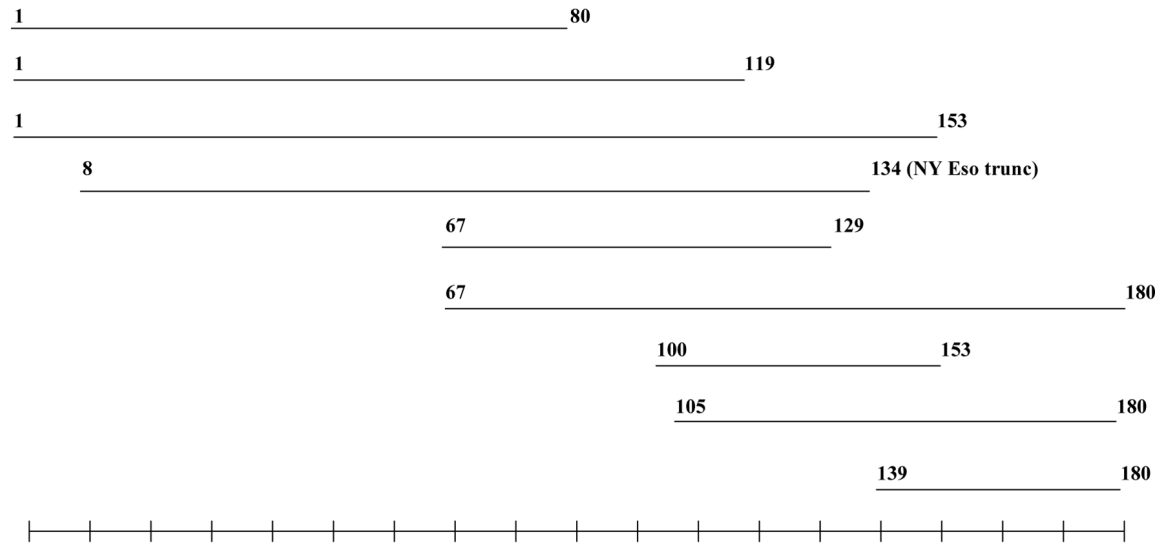

AA 1

180

\section{B}

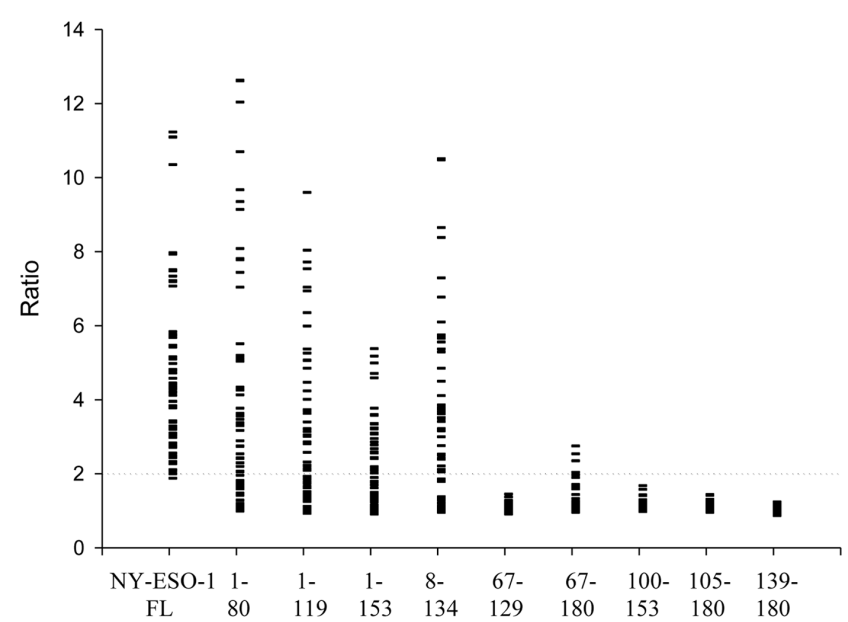

cohorts A, B and C. These cohorts differed regarding the vaccine dose being increased from 10 to $30 \mu \mathrm{g}$ and finally to $100 \mu \mathrm{g}$ of protein plus ISCOM adjuvant.

With respect to the intensity of the serum reaction, we were not able to see a clear correlation to the dose applied. But it became apparent that in the course of repeated vaccinations, antibody titers and cross-reactivity to LAGE $1 \mathrm{~A}$ and B increased as an indicator of an enhanced immune response (Fig. 5).

Eight patients had a pre-existing antibody response against NY-ESO-1 FL. In four of these, the antibody response did not change significantly during the vaccination course whereas the remaining four patients showed an increased immune reaction to NY-ESO1 and an increased cross-reactivity. After the end of the vaccination, we noted a partial decrease of the immune response. But, on the contrary, there were four patients that even showed a remarkable increase in serum reactivity persisting until end of study.

Epitope specificity of NY-ESO-1 restricted immune response. For epitope mapping, sera with positive responses against NY-ESO-1 FL where further analyzed for binding specificity

Figure 4. Patient serum antibody responses against NY-ESO-1-fragments (A) NY-ESO-1-fragments. NY-ESO-1 FL = AA 1-180; various fragments with the respective AA-residues are depicted. (B) Antibody responses against NY-ESO-1 FL and NY-ESO-1-fragments. Y-axis, ratio of flow cytometry g-mean values of antigen expressing to empty vector expressing yeast cells. Each dash represents the ratio of an individual serum staining on the indicated antigen expressing yeast cells. Values below the threshold 2 (dotted line) were considered negative.

to various NY-ESO-1 fragments: 1-80, 1-119, 1-153, 8-134 (= truncated), 67-129, 67-180, 100-153, 105-180 and 139-180 (Fig. 4). The correct expression of the fragments was shown by Western blotting and flow cytometry with anti-PentaHis-tag antibody in a similar way as depicted in Fig. 1 (data not shown).

We exclusively detected antibody reactions to the $\mathrm{N}$-terminal part of the protein. From the 78 serum samples with positive immune response to NY-ESO-1 FL, 47 showed a reaction with the fragment 1-80. For five of these, this was the only positive reaction with one of the fragments; the remaining showed a positive staining for fragment 1-119 as well. From these 42 serum samples, 40 showed an antibody response against NY-ESO-1 truncated (8-134). Furthermore, 27 samples reacted with the NY-ESO-1 fragment 1-153.

All serum samples of one particular patient (101-MEM) additionally recognized NY-ESO-1 fragment 67-180 (Fig. 4B). This patient was already presenting with a spontaneously pre-existing antibody response against NY-ESO-1 FL and the $\mathrm{N}$-terminal fragments mentioned above but not against the C-terminal fragments except for the 67-180 fragment. There was another patient (129-RJS) with a spontaneous antibody 


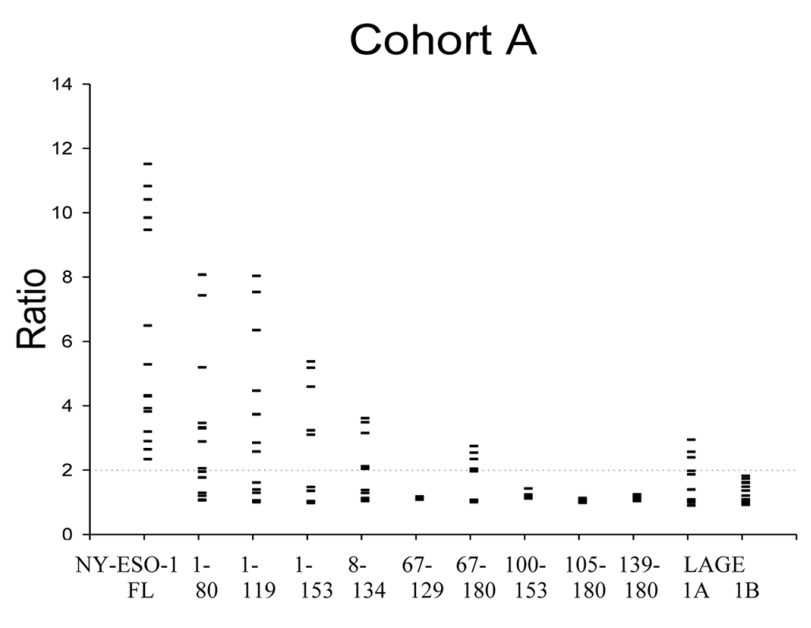

Cohort B

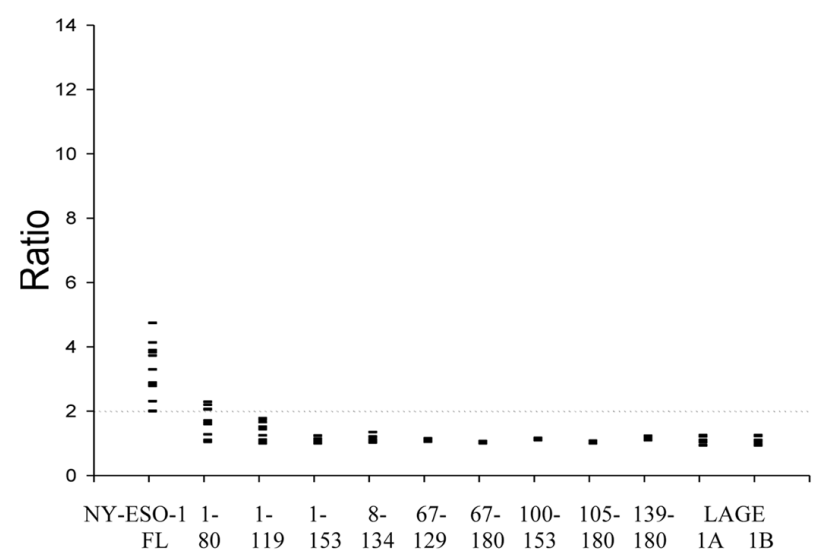

Cohort C

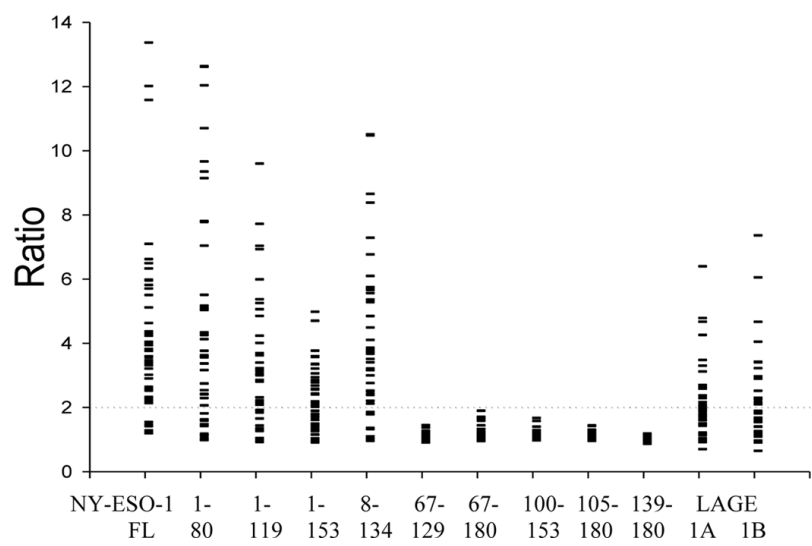

Figure 5. Patient cohorts serum antibody responses against NY-ESO-1fragments and cross-reactivity to LAGE $1 \mathrm{~A}$ and $1 \mathrm{~B}$. Antibody responses of the trial cohorts with ISCOMATRIX (cohort A, B and C) against NY-ESO-1 FL and NY-ESO-1-fragments and LAGE 1A and 1B. Y-axis, ratio of flow cytometry g-mean values of antigen expressing to empty vector expressing yeast cells. Each dash represents the ratio of an individual serum staining on the indicated antigen expressing yeast cells. Values below the threshold 2 (dotted line) were considered negative.

response against NY-ESO-1FL and against the fragments 1-80, 1-119, 1-153, 8-134 (trunc.).

For both patients, this reaction pattern did not change during the vaccination course. All other patients with preexisting spontaneous antibody responses against NY-ESO-1 FL

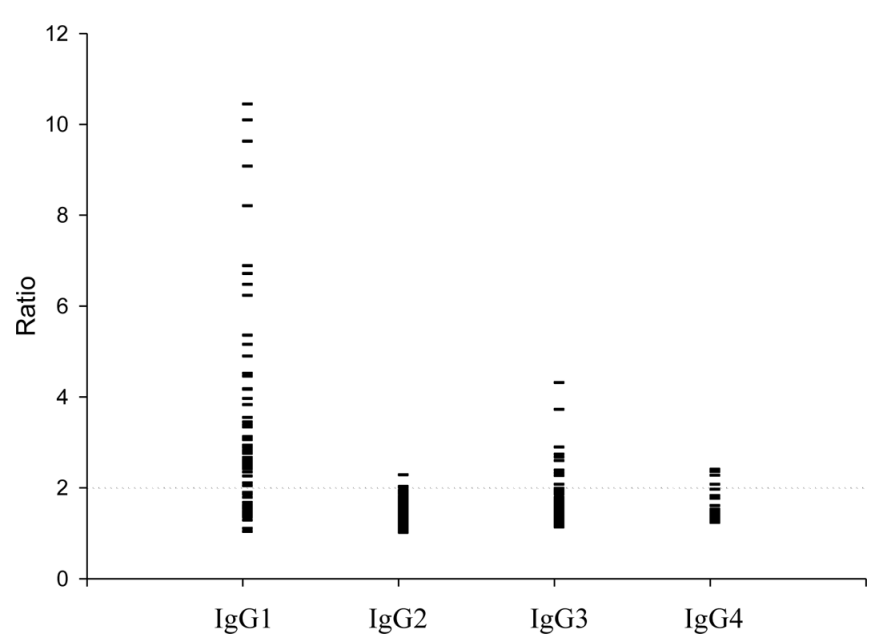

Figure 6. IgG subclass analysis of NY-ESO-1 FL antibody response. Y-axis, ratio of flow cytometry g-mean values of antigen expressing to empty vector expressing yeast cells. Each dash represents the ratio of an individual serum staining on the indicated antigen expressing yeast cells. Values below the threshold 2 (dotted line) were considered negative.

showed during the course of vaccination an antibody reaction against at least one more fragment. No antibody responses at all could be detected against the fragments 67-129, 100-153, 105-180, 139-180.

There was a dose-dependent correlation between the antibody response and the fragment length, which they reacted with: In cohort A (10 $\mu \mathrm{g}$ protein), there was only a reaction detectable against fragment 1-80 and 1-119 (except for the patient 101-MEM with preexisting immune response), in cohort B (30 $\mu$ g protein) only against fragment 1-80 and in the cohort $\mathrm{C}$ (100 $\mu \mathrm{g}$ protein), we found immune responses against the fragments 1-80, 1-119, 8-134 (trunc.) and 1-153 (Fig. 5).

IgG subclasses analysis. Serum samples reacting with NY-ESO-1 FL were additionally subjected to IgG subclass testing (IgG subclass 1-4) to analyze a potential influence of the vaccination on the IgG subclass pattern (Fig. 6). The staining was performed in a similar way as described above but for detection, we used subclass (IgG1, IgG2, IgG3, IgG4)specific secondary antibodies. Again, a serum sample was considered positive, when the ratio of the mean fluorescence intensity (g-mean) of CT-antigen expressing yeast versus empty vector expressing yeast was $>2$.

We predominantly found antibodies from subclass IgG1 in all but one patient. There were four patients with an additional but weaker $\operatorname{IgG} 2$ response and one single patient, who did not have an $\mathrm{IgG} 1$ but an $\mathrm{IgG} 2$ response instead. This patient was the one described above who did not have an immune response to NY-ESO-1 FL. In terms of IgG3 antibody responses, there were altogether 12 positive serum samples from 6 patients. One single patient with pre-existing antibodies against NY-ESO-1 FL and the fragments 1-80, 1-119, 1-153, 8-134 (trunc) and 67-180 showed in addition to his IgG1 response some IgG2-, IgG3 - and IgG4 antibodies. Compared to the IgG1 response that was sometimes strongly positive, the reactions of the other subclasses remained rather weak. 


\section{Discussion}

In this phase I LUD99-008 vaccine trial, we analyzed in detail the humoral immune response against the NY-ESO-1 protein before and after vaccination with ISCOMATRIX using the RAYS technology. During the course of repeated vaccinations with the adjuvant, antibody titers against NY-ESO-1 and cross-reactivity to LAGE $1 \mathrm{~A}$ and $\mathrm{B}$ increased as an indicator of an enhanced immune response, whereas no antibody response could be detected after vaccination without the adjuvant. Our fragment analysis revealed that the humoral response was almost exclusively directed against the $\mathrm{N}$-terminal fragments and the number of fragments and their length being recognized by the NY-ESO-1-specific antibodies was increasing during the course of vaccination. The humoral immune response mainly consisted of antibodies of the IgG1 and IgG3 subclass. We rarely found $\mathrm{IgG} 2$ and $\mathrm{IgG} 4$ subclass antibodies.

For our analysis we used the RAYS system (9-11), which has been shown to be equally sensitive as Western blot analysis or ELISA to detect NY-ESO-1 antibodies and even more sensitive to detect SSX-2 antibody-response (6). The results for NY-ESO-1 antibody responses were similar to the ELISA and Western blot results (17) confirming again the equivalent sensitivity of the RAYS system with respect to NY-ESO-1 antibodies. All sero-positive patients showed a specific cellular immune reaction, except for one patient in the protein cohort with negative serology in ELISA and RAYS, but a cellular response, which was weaker though. All patients receiving a vaccination with ISCOMATRIX adjuvant generated a specific antibody response but none of the protein only or the placebo group. This indicates that this protein-vaccination without an appropriate adjuvant was not able to sufficiently stimulate the immune system to evoke an antibody response. We believe this is important for a full cellular immune reaction to be enhanced as a result of cross-presentation of immune complexes. On the other hand, ISCOMATRIX seems to be a potent adjuvant by increasing antigen presenting capacities of dendritic cells and thereby eliciting broad antibody- as well as potent $\mathrm{CD} 4^{+}$and $\mathrm{CD}^{+} \mathrm{T}$ cell responses $(19,20)$. The increasing reactivity of antibodies was not only apparent by increasing antibody titers during the vaccination course. Moreover, we found an increased number of epitopes that were recognized and an increase of the cross-reactivity with LAGE-1A and B which was clearly dose-dependent. This cross-reactivity is due to the high homology between NY-ESO-1 and LAGE-1 rather than due to epitope spreading. At the end of the vaccination course, we partially realized decreasing antibody titers confirming previous observations that continuous antigen stimulation is necessary to maintain antibody titers against NY-ESO-1 (21-23). Some patients, however, clearly showed a persisting and increasing antibody titer. Further research is needed to clarify the mechanisms responsible for this continuing antibody response. In our NY-ESO-1 fragment analysis, we found the main localization of antibody epitopes located at the N-terminus of the NY-ESO-1 protein, confirming previous results $(16,24)$. There was only one patient in our study, who developed antibodies against the C-terminal fragment (67-180).

The staining of longer fragments always correlated with the staining of the shorter encompassed fragments arguing for mainly non-conformational epitopes. A strong NY-ESO-1 FL staining resulted almost always in detectable fragment reactions whereas serum samples with a weak NY-ESO-1 FL staining rarely showed positive fragment data. We also noted an increase of the immune reaction with the fragments after repeated vaccinations and with increased vaccine dosing (cohort C). In our antibody subclass analysis, nearly all sero-positive patients had developed an IgG1 and, partially, an IgG3 response. In humans, $\operatorname{IgG} 1$ and $\operatorname{IgG} 3$ are the most prominent type 1 reaction IgG subclasses with important functions like opsonization, complement activation and antibody-dependent cellular cytotoxicity (ADCC) (25).

Furthermore, it is known that antibodies play an important role in cross-priming through antibody-mediated crosspresentation (26). Immune-complexes consisting of the antigen and $\mathrm{IgG1}$ or IgG3 mainly bind to Fc $\gamma \mathrm{RIIa}(27,28)$, which is a potent leukocyte activator and can stimulate the release of high levels of inflammatory cytokines. Thus, the isotypes induced by ISCOMATRIX could be effective at inducing dendritic cell activation and maturation leading to an efficient antigen presentation. There was only one patient, who besides the IgG1 response also showed an IgG 4 subtype directed against the C-terminal fragment, which was unusual as well, as indicated above. As this IgG4 response only occurred in one patient we believe that this may be rather an individual event than a typical immune response to the vaccine. There was a previous report about vaccination with recombinant carcino-embryonic antigen (CEA) in patients with colorectal cancer. In that study, patients developed primarily an IgG1 and $\mathrm{IgG} 2$ response and several months later an $\mathrm{IgG} 4$ response. There was even a gradual shift reported from a predominant IgG1 response at 6 months to an IgG4 response at 15 months. This discrepancy compared to our study may be due to different vaccination conditions, e.g. the adjuvant, which was GM-CSF, and maybe the repeated vaccinations over a one year period (29).

In this study, the new adjuvant ISCOMATRIX was used, which is able to stimulate $\mathrm{CD} 4^{+}, \mathrm{CD} 8^{+}$and antibody responses $(17,30)$ and predominantly skews the immune response to a Th1 type (20), thus providing very favorable conditions to elicit a broad and efficient $\mathrm{T}$ cell response. A simple serologic analysis of the humoral immune response is enough for screening, whether the immune system reacted to the vaccine due to the interactions of the cellular and humoral immune system mentioned above. In order to elicit an effective and durable immune response, a vaccine must be able to stimulate a $\mathrm{CD}^{+}$and $\mathrm{CD}^{+} \mathrm{T}$ cell response (31-33). It was also shown that patients with high antibody titers against NY-ESO-1 produce an effective $\mathrm{CD}^{+} \mathrm{T}$ cell response (22). Moreover, a clear correlation of an NY-ESO-1-specific antibody response and NY-ESO-1-specific CD4 ${ }^{+}$T cells was described (34). In summary, an orchestrated interaction of different parts of the immune system seems to be necessary to generate an efficient and durable immune response. This was even further dissected: regarding the timeline, it could be demonstrated that a humoral immune response and a $\mathrm{CD} 4^{+} \mathrm{T}$ cell reaction were preceding a CD8 ${ }^{+}$reaction, suggesting that a sufficient amount of specific antibodies and effective $\mathrm{T}$ cell help could be necessary for effective cross-presentation and -priming of the $\mathrm{CD} 8^{+} \mathrm{T}$ cells (16). Our results argue for the theory that specific antibodies binding the target protein play a major role in protein-immune- 
complex uptake into specified dendritic cells and may thereby greatly contribute to antigen cross-presentation and efficient $\mathrm{CD}^{+} \mathrm{T}$ cell activation (35).

\section{Acknowledgements}

This work was supported in part by HOMFOR, the Ludwig Institute for Cancer Research and the Cancer Research Institute.

\section{References}

1. Baxevanis CN, Perez SA and Papamichail M: Combinatorial treatments including vaccines, chemotherapy and monoclonal antibodies for cancer therapy. Cancer Immunol Immunother 58: 317-324, 2009.

2. Fiszer D and Kurpisz M: Major histocompatibility complex expression on human, male germ cells: a review. Am J Reprod Immunol 40: 172-176, 1998.

3. Scanlan MJ, Simpson AJ and Old LJ: The cancer/testis genes: review, standardization, and commentary. Cancer Immun 4: 1, 2004.

4. Chen YT, Scanlan MJ, Sahin U, et al: A testicular antigen aberrantly expressed in human cancers detected by autologous antibody screening. Proc Natl Acad Sci USA 94: 1914-1918, 1997.

5. Gnjatic S, Nishikawa H, Jungbluth AA, et al: NY-ESO-1: review of an immunogenic tumor antigen. Adv Cancer Res 95: 1-30, 2006.

6. Mischo A, Wadle A, Watzig K, et al: Recombinant antigen expression on yeast surface (RAYS) for the detection of serological immune responses in cancer patients. Cancer Immun 3: $5,2003$.

7. Boder ET and Wittrup KD: Yeast surface display for directed evolution of protein expression, affinity, and stability. Methods Enzymol 328: 430-444, 2000.

8. Boder ET and Wittrup KD: Yeast surface display for screening combinatorial polypeptide libraries. Nat Biotechnol 15: 553-557, 1997.

9. Wadle A, Mischo A, Imig J, et al: Serological identification of breast cancer-related antigens from a Saccharomyces cerevisiae surface display library. Int J Cancer 117: 104-113, 2005.

10. Wadle A, Kubuschok B, Imig J, et al: Serological immune response to cancer testis antigens in patients with pancreatic cancer. Int J Cancer 119: 117-125, 2006.

11. Mischo A, Kubuschok B, Ertan K, et al: Prospective study on the expression of cancer testis genes and antibody responses in 100 consecutive patients with primary breast cancer. Int J Cancer 118: 696-703, 2006.

12. Kim MS, Choi HY, Choi YS, Kim J and Kim YS: Optimized serological isolation of lung-cancer-associated antigens from a yeast surface-expressed cDNA library. J Microbiol Biotechnol 17: 993-1001, 2007.

13. Disis ML, Calenoff E, McLaughlin G, et al: Existent T-cell and antibody immunity to HER-2/neu protein in patients with breast cancer. Cancer Res 54: 16-20, 1994.

14. Jager E, Chen YT, Drijfhout JW, et al: Simultaneous humoral and cellular immune response against cancer-testis antigen NY-ESO-1: definition of human histocompatibility leukocyte antigen (HLA)-A2-binding peptide epitopes. J Exp Med 187: 265-270, 1998

15. Dhodapkar KM, Krasovsky J, Williamson B and Dhodapkar MV: Antitumor monoclonal antibodies enhance cross-presentation ofcCellular antigens and the generation of myeloma-specific killer T cells by dendritic cells. J Exp Med 195: 125-133, 2002.

16. Valmori D, Souleimanian NE, Tosello V, et al: Vaccination with NY-ESO-1 protein and $\mathrm{CpG}$ in Montanide induces integrated antibody/Th1 responses and CD8 T cells through cross-priming. Proc Natl Acad Sci USA 104: 8947-8952, 2007.
17. Davis ID, Chen W, Jackson H, et al: Recombinant NY-ESO-1 protein with ISCOMATRIX adjuvant induces broad integrated antibody and CD4(+) and CD8(+) T cell responses in humans. Proc Natl Acad Sci USA 101: 10697-10702, 2004.

18. Sahin U, Tureci O, Schmitt H, et al: Human neoplasms elicit multiple specific immune responses in the autologous host. Proc Natl Acad Sci USA 92: 11810-11813, 1995.

19. Sjolander A, Ahlborg N, Stahl S and Andersson R: Characterization of immune responses to experimental polyvalent subunit vaccines assembled in iscoms. Mol Immunol 35: 159-166, 1998.

20. Maraskovsky E, Sjolander S, Drane DP, et al: NY-ESO-1 protein formulated in ISCOMATRIX adjuvant is a potent anticancer vaccine inducing both humoral and $\mathrm{CD} 8^{+} \mathrm{t}$-cell-mediated immunity and protection against NY-ESO- $1^{+}$tumors. Clin Cancer Res 10: 2879-2890, 2004.

21. Jager E, Stockert E, Zidianakis Z, et al: Humoral immune responses of cancer patients against cancer-testis antigen NY-ESO-1: correlation with clinical events. Int J Cancer 84 : 506-510, 1999.

22. Jager E, Nagata Y, Gnjatic S, et al: Monitoring CD8 T cell responses to NY-ESO-1: correlation of humoral and cellular immune responses. Proc Natl Acad Sci USA 97: 4760-4765, 2000.

23. Stockert E, Jager E, Chen YT, et al: A survey of the humoral immune response of cancer patients to a panel of human tumor antigens. J Exp Med 187: 1349-1354, 1998.

24. Valmori D, Souleimanian NE, Hesdorffer CS, Ritter G, Old LJ and Ayyoub M: Identification of B cell epitopes recognized by antibodies specific for the tumor antigen NY-ESO-1 in cancer patients with spontaneous immune responses. Clin Immunol 117: 24-30, 2005.

25. Papadea $\mathrm{C}$ and Check IJ: Human immunoglobulin G and immunoglobulin $\mathrm{G}$ subclasses: biochemical, genetic, and clinical aspects. Crit Rev Clin Lab Sci 27: 27-58, 1989.

26. Regnault A, Lankar D, Lacabanne V, et al: Fcgamma receptormediated induction of dendritic cell maturation and major histocompatibility complex class I-restricted antigen presentation after immune complex internalization. J Exp Med 189: 371-380, 1999.

27. van de Winkel JG and Capel PJ: Human IgG Fc receptor heterogeneity: molecular aspects and clinical implications. Immunol Today 14: 215-221, 1993

28. Houghton AN and Scheinberg DA: Monoclonal antibody therapies-a 'constant' threat to cancer. Nat Med 6: 373-374, 2000.

29. Ullenhag GJ, Frodin JE, Strigard K, Mellstedt H and Magnusson CG: Induction of IgG subclass responses in colorectal carcinoma patients vaccinated with recombinant carcinoembryonic antigen. Cancer Res 62: 1364-1369, 2002.

30. Chen $\mathrm{Q}$, Jackson $\mathrm{H}$, Parente $\mathrm{P}$, et al: Immunodominant $\mathrm{CD}^{+}$ responses identified in a patient vaccinated with full-length NY-ESO-1 formulated with ISCOMATRIX adjuvant. Proc Natl Acad Sci USA 101: 9363-9368, 2004.

31. Williams MA, Holmes BJ, Sun JC and Bevan MJ: Developing and maintaining protective $\mathrm{CD} 8^{+}$memory $\mathrm{T}$ cells. Immunol Rev 211: 146-153, 2006.

32. Ridge JP, Di Rosa F and Matzinger P: A conditioned dendritic cell can be a temporal bridge between a $\mathrm{CD}^{+} \mathrm{T}$-helper and a T-killer cell. Nature 393: 474-478, 1998.

33. Bourgeois C, Rocha B and Tanchot C: A role for CD40 expression on $\mathrm{CD}^{+} \mathrm{T}$ cells in the generation of $\mathrm{CD}^{+} \mathrm{T}$ cell memory. Science 297: 2060-2063, 2002.

34. Gnjatic S, Atanackovic D, Jager E, et al: Survey of naturally occurring $\mathrm{CD}^{+} \mathrm{T}$ cell responses against NY-ESO-1 in cancer patients: correlation with antibody responses. Proc Natl Acad Sci USA 100: 8862-8867, 2003.

35. Nagata Y, Ono S, Matsuo M, et al: Differential presentation of a soluble exogenous tumor antigen, NY-ESO-1, by distinct human dendritic cell populations. Proc Natl Acad Sci USA 99: 10629-10634, 2002. 